Relations industrielles

Industrial Relations

\title{
Dans l'administration d'un décret
}

Rôles respectifs du secrétaire et de l'inspecteur du comité paritaire

\section{J.-Médard Ouellet}

Volume 3, numéro 2, octobre 1947

URI : https://id.erudit.org/iderudit/1023568ar

DOI : https://doi.org/10.7202/1023568ar

Aller au sommaire du numéro

\section{Éditeur(s)}

Département des relations industrielles de l’Université Laval

ISSN

0034-379X (imprimé)

1703-8138 (numérique)

Découvrir la revue

Citer cet article

Ouellet, J.-M. (1947). Dans l'administration d'un décret : rôles respectifs du secrétaire et de l'inspecteur du comité paritaire. Relations industrielles /

Industrial Relations, 3(2), 28-30. https://doi.org/10.7202/1023568ar

Tous droits réservés (C Département des relations industrielles de l’Université Laval, 1947
Ce document est protégé par la loi sur le droit d'auteur. L’utilisation des services d'Érudit (y compris la reproduction) est assujettie à sa politique d'utilisation que vous pouvez consulter en ligne.

https://apropos.erudit.org/fr/usagers/politique-dutilisation/ 


\title{
Dans l'administration d'un décret
}

\section{Rôles respectifs du secrétaire et de l'inspecteur du comité paritaire}

\author{
J.-Médard OUELLET
}

Les fonctions de secrétaire et d'inspecteur sont de la plus haute importance dans l'administration d'un décret par le Comité Paritaire.

Nous savons tous qu'un décret-loi faisant l'objet d'un arrêté ministériel sanctionné en vertu de la Loi de la Convention Collective est confié à cette chambre corporative qu'on appelle comité paritaire qui doit l'appliquer à tous les assujettis, viz. aux contractants par convention et aux tiers par extension.

Les membres d'un Comité Paritaire étant nommé par les parties contractantes sont chargés de surveiller l'application du décret et leurs décisions doivent être prises en assemblées régulières ou spéciales. Or que se passe-t-il en dehors des assemblées? C'est ici qu'entre en jeu la fonction la plus importante du secrétaire.

Va sans dire que celui-ci est lié par toutes décisions prises par les membres en assemblée et son premier rôle consiste à les exécuter à la lettre. Mais il y a bien des manières d'exécuter et c'est ici qu'un bon secrétaire doit faire preuve de beaucoup de tact. Une loi est toujours désagréable à suivre parce que toute règlementation, la meilleure soitelle, comporte des entraves à la liberté individuelle pour pouvoir assurer le bien commun. Or il arrive que les membres d'un tel comité qui ont à prendre des décisions, envisagent cette dernière hypothèse que la loi doit s'appliquer à tous pour que le bien commun en résulte. Le secrétaire qui a à régler tel problène avec tel individu se doit d'en considérer toutes les données afin d'orienter les décisions qu'il aura ensuite charge d'appliquer. Il doit certes faire preuve d'objectivité d'abord mais en homme intelligent, il ne doit pas oublier la subjectivité d'un problème dont chaque nuance doit être habilement démontrée aux membres pour que ceux-ci puissent rendre une décision juste. Pour qu'elle le soit, la légalité n'est pas toujours suffi sante; il y faut bien souvent l'équité.

Le secrétaire, conscient de ses responsabilités, se doit d'étudier à fond et dans cet esprit, tout problème à être soumis au comité dont les membres ne peuvent en être saisis qu'à l'assemblée. Ceci a aussi pour effet de simplifier la tenue des réunions qui sont toujours trop longues. En passant, qu'il nous soit permis de rendre hommage aux officiers et membres des différents Comités Paritaires de cette province qui sacrifient de leur temps pour le bien de la cause, car les nombreuses assemblées les réclament beaucoup plus pour l'avancement de la profession et la sécurité ouvrière que pour leur bien personnel.

Après avoir étudié chaque cas et les avoir soumis aux membres, le secrétaire doit exécuter leurs résolutions, avons-nous dit. Encore là, il faut beaucoup de souplesse pour faire agréer une décision, qui souvent ne rencontre pas les vues de l'intéressé. Il ne suffit pas pour le secrétaire de servir d'intermédiaire passif entre le Comité et les assujettis, mais il faut que son rôle soit positif et qu'il fasse comprendre aux parties en cause que la décision rendue ne pouvait l'être autrement sans léser les droits de quelqu'un. Il faut en un mot, qu'il fasse fonction de négociateur entre les assujettis et le Comité tout en restant lié aux décisions de celui-ci sans léser les droits de ceux-là, autant que faire se peut.

Le secrétaire de Comité Paritaire doit être impartial et discret dans ses relations avec les assujettis, employeurs ou employés. Ayant à traiter avec les deux, il ne doit avoir comme parti pris, que la justice, l'équité et comme norme interprétative, que le décret qu'il a en mains et les lois qui le conditionnent. Il faut qu'il connaisse autant l'esprit que la lettre de ces lois pour pouvoir exercer un jugement sain, et les personnes physiques ou juridiques visées par tel décret n'en tireront un bénéfice réel que si le secrétaire, par une psychologie de bon aloi, sait le rendre populaire. Ainsi, étant en contact fréquent avec ces personnes, il lui faut répandre l'idée du bien fondé d'une telle règlementation, même extensionnée aux tiers car les objections viennent le plus souvent de ces derniers, cela s'explique.

Ayant démontré les aptitudes requises d'un bon secrétaire, voyons maintenant quelle est sa fonction. C'est lui qui tient les minutes des assemblées, en dresse le procès-verbal qu'il soumet; pour approbation à une assemblée subséquente, reçoit les communications adressées au Comité paritaire, institue et signe, au nom du Comité, toutes les procédures que pourrait requérir la violation du décret et des règlements dudit Comité, expédie toute la correspondance, et en outre, exerce tous autres pouvoirs qui lui sont conférés par la Loi. 
Ainsi au paragraphe e) de l'Art. 20 de la Loi de la Convention Collective il est stipulé que « le secrétaire et l'inspecteur peuvent de droit et en tout temps examiner le système d'enregistrement, le régistre obligatoire et la liste de paye de tout employeur, en prendre des copies ou extraits, vérifier auprès de tout employeur et de tout salarié le taux du salaire, la durée du travail, le régime d'apprentissage et l'observance des autres dispositions du décret, requérir même sous serment et privément de tout employeur ou de tout salarié, et même au lieu du travail, les renseignements jugés nécessaires, et, tels renseignements étant consignés par écrit, exiger la signature de l'intéressé ».

Le secrétaire doit aussi surveiller l'application des règlements du Comité, et comme il en est, pour ainsi dire, la cheville ouvrière, un bon secrétaire doit faire des suggestions au Comité en vue d'en amender les clauses s'il y a lieu pour sa bonnc administration. Il doit en faire autant pour le décret lui-même ainsi que pour la partie administrative de la Loi de la Convention Collective décrite dans ce Bulletin (No. de Juin 1947) comme suit: Rôle du Comité Paritaire, sa composition, sa formation, ses droits, ses obligations, son extinction, la qualification des salariés, la violation de la loi, les pénalités et les procédures.

En plus d'être trésorier, le secrétaire d'un Comité Paritaire assez important est aussi un gérant parce qu'il a la surveillance d'un bureau où il $\mathrm{y}$ a des employés à diriger. Il doit donc avoir les qualités d'un bon patron tout en appliquant la discipline nécessaire au bon fonctionnement du secrétariat.

Enfin pour l'information des membres du Comité, le secrétaire doit être vigilent et très expéditif dans son travail et prendre des initiatives comme celles de compiler des, statistiques, surtout pour les décrets applicables à l'industrie, et suivre de très près l'évolution du champ d'activité économique couvert par son décret. Pour cela il lui faut lire et étudier beaucoup mais surtout observer le milieu social où l'on vit.

Comme on le voit, un tel secrétaire fait plus que la fonction que réserve à ce terme la définition du dictionnaire, mais il est sans doute limité dans ses pouvoirs tout comme l'inspecteur d'un Comité Paritaire. Avant de délimiter leurs pouvoirs, voyons en quoi consiste le rôle de l'inspecteur.

Celui-ci est aussi essentiel au Comité que celui-là et leurs fonctions sont complémentaires. Tous deux contribuent au progrès d'une profession donnée. Le comité paritaire lui-même est le pivot de ce progrès, sa raison d'être se trouvant dans la volonté des parties contractantes.
C'est donc dire que cet axe est essentiel à la marche du progrès social dans le domaine des relations ouvrières.

Voyons maintenant quel rôle l'inspecteur joue exactement. Disons d'abord que sa fonction semble ingrate, précisément parce qu'il est un enquêteur qui arrive au moment où l'on s'y attend le moins chez un employeur, pour exiger qu'on lui fasse voir les bordereaux de paye, les régistres de salaires et les feuilles de temps des employés. Ceci constitue un excellent moyen pour le comité paritaire de se rendre impopulaire, et pour l'inspecteur de se faire aimer dans le mauvais sens, si celui-ci n'est pas un homme affable, poli, discret et surtout possédant une notion exacte de ce mot d'ordre: 《se mêler de ses affaires».

L'inspecteur doit connaître autant que possible l'industrie ou le commerce régi par le décret et ne pas être un profane des catégories de professions ou métiers également visés.

Son premier devoir est de rapporter fidèlement au secrétaire le fruit de ses enquêtes et d'en disséquer la teneur afin de découvrir les infractions au décret s'il s'en trouve. Le secrétaire voit ensuite s'il y a matière à réclamation en faveur de la personne lésée dans ses droits ou si le cas doit être référé au Comité pour étude.

Le devoir secondaire de l'inspecteur est de percevoir les dus qu'il remet au secrétaire, s'il est requis de le faire par le Comité. Il faut dire, ici, que cette tâche n'incombe pas à tous les inspecteurs, le prélèvement de certains comités se payant directement par chèque ou autrement au bureau.

Un devoir auquel nul inspecteur ne peut se soustraire est d'entendre les plaintes à lui soumises, par quiconque est assujetti au décret. Il doit les approfondir avant de les soumettre au secrétaire et s'il y a divergence d'opinion dans la version de l'employeur et de l'employé, le secrétaire soumettra le litige au Comité qui décidera de l'attitude à suivre. Il doit, en plus, surveiller l'apprentissage s'il en est requis.

Nous avons vu un peu jusqu'où s'étendent les pouvoirs de l'un et de l'autre, mais complétons en disant que le secrétaire ne peut pas renverser une décision du Comité, ni passer outre; qu'il lui est interdit de déroger aux règlements que s'est donnés le Comité, quant aux procédures judiciaires, aux déboursés d'argent ou à la régie interne où ses pouvoirs sont bien déterminés. Le sont également ceux de l'inspecteur et il faut ajouter que celui-ci étant en relations avec employeurs et employés, il ne doit jamais se permettre, dans l'exercice de ses fonctions, de faire allusion à autre chose que ce qui fait l'objet de son enquête. Par ex- 
emple, il ne doit pas profiter de son contrat pour solliciter les employés pour telle union de son choix ou contre telle autre à l'endroit de laquelle il nourrit des préjugés.

Enfin, nous n'insisterons jamais trop, les deux ne doivent pas commettre d'indiscrétion, le secret professionnel étant un de leur plus précieux ressorts, avec celui de la conscience professionnelle pour leur avancement comme pour leur réputation. Unis et conscients de leur rôle, ces deux auxiliaires précieux serviront la cause du bien commun dont celui de la profession a été confié au Comité paritaire par l'Etat, sur demande des parties représentant les employeurs et les employés.

Pour terminer, émettons le voeu que ces travailleurs sociaux puissent tous posséder les notions fondamentales de sociologie et d'économie politique et sur les solutions apportées par la morale aux grands problèmes sociaux et économiques de l'heure présente. Au moins, ils doivent suivre le développement parallèle des organisations ouvrières et patronales, les revendications des salariés et la mission sociale de l'Etat.

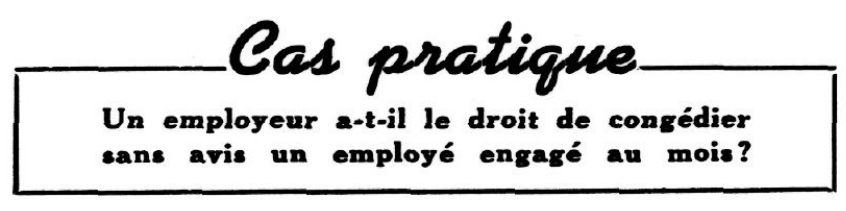

\section{Donat QUIMPER}

La réponse directe à cette question dépend de l'existence ou non de règlements municipaux à ce sujet dans la localité où travaille l'employé; et de l'application possible de la loi des Muîtres et des serviteurs.

En effet, depuis l'abrogation des règlements du service sélectif qui jusqu'au 31 mars 1947 imposaient en théorie, un avis de séparation de $\vec{\imath}$ jours, les relations entre patrons et employés ne sont plus désormais assujetties qu'aux prescriptions de la loi ordinaire. Or le code civil, bien que ne comportant rien d'explicite sur les avis de congédiement et d'abandon de service, indique cependant (art. 1670) que les droits et obligations résultant du bail de service personnel sont régis sous certains rapports, dans les campagnes, par une loi spéciale, et dans les villes et villages, par les règlements des conseils municipaux.

Conséquemment, lorsqu'il s'agit de répondre à une question comme celle-ci, ce sont d'abord les règlements municipaux régissant les relations entre patrons et employés qu'il faut consulter. Là où il n'existe pas de tels règlements, il faut référer à la loi spéciale indiquée par le code civil et contenue dans nos statuts sous le titre de: Loi des Maîtres et des serviteurs. (S.R.Q. 1941, c. 328). Cette loi est désuète comme les expression du titre qu'elle porte. Le mot Maîtres, en effet, n'a pas dans cette loi le sens restreint qu'on serait porté à lui donner à première vue, pas plus que le mot serviteurs n'a le sens exclusif de domestique. Il faut donner à ces expressions le sens qu'on donne aujourd'hui aux mots patrons et employés.

$\mathrm{Au}$ cours de son demi-siècle d'existence, cette loi n’a été appliquée que très rarement à cause des régions et territoires qu'elle excepte, et à cause des catégories d'employés qu'elle exclut. En effet, elle ne s'applique pas aux employés engagés à la pièce, à l'heure ou à l'entreprise. Elle ne s'applique pas aux cités de Québec et de Montréal, ni aux autres cités constituées en corporation, ni aux villes et villages qui ont passé ou qui pourront passer des règlements régissant les relations entre maîtres et serviteurs. C'est donc une loi supplétive qui ne s'applique qu'en autant et aussi longtemps que le champ n'est pas occupé par des règlements municipaux.

Au surplus, croyons-nous devoir ajouter que sur la question spéciale du droit de faire des congédiements pour cause, cette loi nous paraît venir en conflit avec l'article 21 de la loi de relations ouvrières. (S.R.Q. 1941, c. 162-A). A tout événement, puisqu'elle existe dans les statuts et demeure ainsi susceptible d'application, nous citons les quelques articles qui peuvent répondre à la présente question:-

«Tout domestique, serviteur, compagnon ou journalier, engagé à la semaine, au mois ou à l'année, et non à la pièce ou à l'entreprise, ou pour une période fixe, qui a l'intention d'abandonner le service pour lequel il est engagé à l'expiration de son engagement, doit donner au moins une semaine d'avis de cette intention lorsque son engagement est à la semaine, ou deux semaines si son engagement est au mois, et un mois lorsque l'engagement est à l'année »... (art. 3).

Cette obligation est réciproque. «Le maître et la maîtresse ou le patron doit donner un pareil avis à tout serviteur, compagnon ou journalier, engagé à la semaine, au mois ou à l'année, dont les services ne sont plus requis; mais tout domestique, serviteur, compagnon ou journalier, ainsi engagé peut être congédié à l'expiration de son engagement ou avant, sans avis, en lui payant le plein montant des gages auquel il aurait eu droit à l'expiration de son engagement et si l'avis requis lui avait été donné». (art. 4). 